\title{
18
}

\section{Experience in Application of Composable Security Properties}

\author{
Qi Shi \\ School of Computing \& Mathematics \\ Liverpool John Moores University \\ Byrom Building, Byrom Street \\ Liverpool, L3 3AF, U.K.
}

\author{
N. Zhang \\ Department of Computing \\ Manchester Metropolitan University \\ John Dalton Building, Chester Street \\ Manchester, M1 5GD, U.K.
}

\begin{abstract}
Current composable security properties provide a method for designing and evaluating secure systems based on modules. In this paper we first classify these composable properties, and compare their advantages and disadvantages. We then discuss how to apply the composable properties to deal with composition of modules in a cost-effective manner, in order to make use of their advantages and to eliminate or reduce their disadvantages. We finally summarise the lessons we learned from application of the composable properties, with regard to the issues of system functionality, performance, modularisation, security and costs.
\end{abstract}

\section{INTRODUCTION}

In designing and analysing the structure and security of a large system, one often wishes to break the system into simpler modules that can be constructed separately and verified more easily. To apply such a modular approach to developing a secure system, we need a method to cope with composition of modules so as to determine the system's security properties, usually in terms of information flow, from the properties of its modules.

The issues related to composition of modules or components have been addressed in the literature [1-6,8-11]. The theories developed in this field have provided a variety of important insights into composable security properties. Usually a composable property such as restrictiveness [3] states that if it is satisfied by each of the modules, then the property is also satisfied by their overall system. This shows that security properties of the system are determined by means of those of its modules, and hence the security analysis of the system is simplified.

In this paper we first classify composable properties with regard to the extent to which these properties are dependent on interactions among modules. As we will introduce, some composable properties are developed based mainly on internal operations of a module by assuming the worst scenario of its interactions with others, whereas other composable properties are achieved by taking into account both the internal operations of a module and its interactions with others.

We then compare different classes of composable properties in terms of security issues of system development in a modular way. As will be addressed later, each of these composable properties has some advantages but also disadvantages. For instance, the implementation of some 
composable property is easier, but it imposes over-strong security requirements on modules. And the implementation of another composable property is more complicated, but it enforces more appropriate security requirements on modules.

Based on this comparison, we come to discuss how to apply different classes of composable properties in a cost-effective manner, in order to retain their good features and to reduce their bad ones. To do so, a mixed version of different composable properties is probably more effective and desirable. That is, security properties of different modules are analysed by different composable properties. Obviously these mixed composable properties must be consistent in the sense that if each module meets its security property, then their overall system satisfies the system security property.

We finally describe the lessons learned from application of composable properties, with regard to the issues of system functionality, performance, modularisation, security and costs. This addresses some of the problems resulting from development of secure systems and application of composable properties, and then proposes possible solutions to the problems so as to produce useful and reasonably secure systems.

The rest of the paper is organised as follows. Section 2 classifies composable security properties. Security issues of system development in a modular way are addressed in Section 3. Based on these issues, different classes of composable properties are compared in Section 4. The issue of how to apply composable properties cost-effectively is discussed in Section 5. The lessons we learned from application of the composable properties are summarised in Section 6. Finally our conclusions are outlined in Section 7.

\section{COMPOSABLE SECURITY PROPERTIES}

In this section we describe major features of different composable properties which are used to deal with composition of modules. To do so, these properties need to be divided into the following three classes:

- Independent: A composable property of this class is specified based mainly on internal operations of a module without considering its interactions with others. Two major features of such a composable property are composability and separability. The property is composable if it guarantees that a system can satisfy the property, provided each of its modules meets the property. And the property is separable if each of the modules can be separately assessed in terms of the property, i.e. the security of the module is analysed without knowing operational details of other modules. Obviously such a composable property needs to impose security constraints on all input-output entities of every module. The properties presented in $[1-3,5,10]$ belong to this class.

- Dependent: A composable property of this class is developed by taking into account the connectivity of a module with others. That is, the security analysis of a module in terms of this property is dependent on not only internal operations of the module but also its interactions with others. Similarly to the independent class, this composable property requires different modules to possess the same security property.

A major reason for considering interactions is that weak security constraints can be placed on modules without compromising their system security. This is because when the modules are 
composed into a system, only some input-output entities of the modules become those of the system, and others are hidden inside the system. Clearly these hidden entities are unnecessarily demanded to meet security constraints. As a result, security properties of the modules can be adequately weakened.

This composable property might lose separability at first glance since interactions among modules are taken into account. In effect, its separability can still be achieved by properly handling interactions among modules. An example of such a composable property is described in [7].

- Strongly dependent: A composable property of this class is also defined with regard to the connectivity of a module with others. The difference from the other two classes is that it allows different modules to have different security properties so long as their composition leads to a secure system. Thus appropriate security requirements can be enforced on modules. Security analysis of a module in terms of this property is strongly dependent on its interactions with others. Obviously this composable property loses separability. An example of such a composable property is presented in [6].

For further information on the classes of composable properties, the reader may refer to the references attached to this paper.

The independent and dependent classes of composable properties can be used to deal with the case where secure modules are composed into a secure system. In fact, these properties describe a bottom-up method for handling the system security. The strongly dependent class of composable property can be applied to handle two cases. The first is the case in which secure or insecure modules are composed into a secure system, while the second is the case where a secure system can be decomposed into secure or insecure modules. This means that the composable property can be used in both bottom-up and top-down ways. The top-down way is particularly useful for appropriately decomposing a system security requirement into modular security requirements.

\section{SECURITY ISSUES}

In this section we raise some security issues of system development in a modular way, and then compare the three classes of composable properties with regard to these issues in the next section. For simplicity, in this paper we assume that input and output entities in a system only have two security level assignments, low and high.

\subsection{System Decomposition}

As indicated in Section 2, some composable properties describe a bottom-up method, and others introduce a top-down way, for security analysis. This implies that the determination of system security in terms of the composable properties is dependent on system decomposition. In other words, if the system is decomposed in different ways, security analysis could reach different conclusions although the same composable property is used, i.e. the system satisfies the property in terms of some ways of decomposition, but not others. This issue can be illustrated clearly by means of the example given in [12]. 
Consider a system $S$ that has a low output entity $l_{o}$, a high input entity $h_{i}$ and output entity $h_{o}$, and two state entities $K_{1}$ and $K_{2}$. The entity $h_{i}$ has three possible inputs $\{0,1, q\}$, and values of the other entities are either 0 or 1 . The operation of the system is described as follows:

if $h_{i}$ receives a value 0 or 1 , then

$h_{o}$ outputs a value 0

$l_{o}$ outputs a value $h_{i} \oplus K_{1}(\oplus$ stands for xor)

$K_{1}$ and $K_{2}$ are randomly and independently updated

if $h_{i}$ receives value $q$, then

$h_{o}$ outputs the value of $K_{1}$

$l_{o}$ outputs the value of $K_{2}$

$K_{2}$ is updated randomly $\left(K_{1}\right.$ remains unchanged)

Suppose that the composable property of nondeducibility introduced in [10] is used to measure system security. Nondeducibility states that if a low user of a system sees the low input and output sequence of a trace which is a sequence of inputs and outputs as well as states, the low user can also see the same sequence when the high input sequence of the trace is replaced by any possible high input sequence. In addition, the property requires that the system be input total, i.e. no matter what state the system is in, it can accept an input. The composable property states that if every module is nondeducible and input total, then their system is also nondeducible.

It is easy to see that system $S$ satisfies this property. We now decompose the system into two modules in two different ways. One may argue that the system should not be decomposed into modules, because it is quite small. In fact, this system is used just as a simple example to demonstrate a problem which also happens in large systems.

First of all, the system is represented as a composition of two modules $M_{1}$ and $M_{2}$ whose connections are shown in Fig. 1 where an unbroken line denotes a low output and broken lines signify high inputs and outputs. Operations of these modules are defined below:

$$
\begin{aligned}
& M_{1} \text { : if } h_{i} \text { receives a value } 0 \text { or } 1 \text {, then } \\
& h_{o} \text { outputs } 0 \\
& h_{o}^{\prime} \text { outputs } h_{i} \oplus K_{1} \\
& K_{1} \text { is updated randomly } \\
& \text { if } h_{i} \text { receives value } q \text {, then } \\
& h_{o} \text { outputs the value of } K_{1} \\
& h_{o}^{\prime} \text { outputs } q \\
& M_{2} \text { : if } h_{i}^{\prime} \text { receives a value } 0 \text { or } 1 \text {, then } \\
& l_{o} \text { outputs the value of } h_{i}^{\prime} \\
& \text { if } h_{i}^{\prime} \text { receives value } q \text {, then } \\
& l_{o} \text { outputs the value of } K_{2} \\
& K_{2} \text { is updated randomly }
\end{aligned}
$$

It is evident that module $M_{2}$ does not meet nondeducibility since low users can certainly deduce, by obtaining an output 1 , that $h_{i}^{\prime}$ does not receive value 0 . That is, $M_{2}$ does not satisfy the composable property of nondeducibility. So the system composed of these two modules is rejected. 


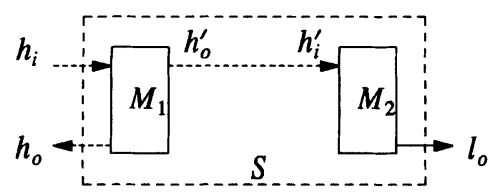

Figure 1

We now discuss the second case of system decomposition, where connections of two modules $M_{1}^{\prime}$ and $M_{2}^{\prime}$ are the same as those of $M_{1}$ and $M_{2}$. Operations of these two modules are specified as follows:

$M_{1}^{\prime}$ : if $h_{i}$ receives a value 0 or 1 , then

$h_{o}$ outputs 0

$h_{o}^{\prime}$ outputs the value of $h_{i}$

if $h_{i}$ receives value $q$, then

$h_{o}$ outputs the value of $K_{1}$

$h_{o}^{\prime}$ outputs $q$
$M_{2}^{\prime}$ : if $h_{i}^{\prime}$ receives a value 0 or 1 , then

$l_{o}$ outputs $h_{i}^{\prime} \oplus K_{1}$

$K_{1}$ and $K_{2}$ are updated

(randomly and independently)

if $h_{i}^{\prime}$ receives value $q$, then

$l_{o}$ outputs the value of $K_{2}$

$K_{2}$ is updated randomly

It is obvious that both of these modules meet the composable property of nondeducibility. Thus the system made up of the modules is accepted by this composable property.

This example indicates that different decompositions of the same system lead to different conclusions of system security by using the same composable property. This raises the issue of how closely the three classes of composable properties are dependent on system decomposition.

\subsection{Strength of Security Requirements}

In practice, the development of a secure system has to take into account other factors such as system functionality and performance. Sometimes, the security requirement of the system restricts the realisation of its function and performance specifications. Consequently, reasonable trade-offs between system security and functionality or performance have to be made in order to produce a useful and adequately secure system. This implies that some relatively weak security requirement needs to be enforced on the system. We believe that many practical security requirements are of this form.

A similar argument is also applied to modular security requirements. That is, weaker security requirements should be placed on modules as long as their composition results in a secure system. This can avoid the unnecessary loss of modular functionality caused by over-strong security requirements. For instance, though module $M_{2}$ in the example given in Section 3.1 does not meet nondeducibility, it can be considered to be secure since its composition with module $M_{1}$ still enables system $S$ to meet the nondeducibility property. This raises the issue of whether or not the three classes of composable properties are able to enforce security requirements of various strength. 


\subsection{Costs of Security Evaluation}

The cost of security evaluation is one of major concerns in the development of secure systems, since it is usually expensive. As introduced earlier, some composable properties are produced by taking into account interconnections among modules, but others not. This means that for some composable property, the security of a module is determined based solely on its internal operations, but for another, it is decided based on the internal operations of the module in conjunction with those of other modules. Intuitively implementation of different composable properties requires different amounts of effort.

Additionally the security evaluation of a module sometimes needs to be repeated due to some errors being found or its functional specification being changed. Such re-evaluation could have impacts on security properties of other modules. To what extent this re-evaluation influences security properties of the other modules is very much dependent on which composable property is applied. This means that applications of different composable properties spend different amounts of effort on security re-evaluation. This analysis raises the issue of how cost-effective the classes of composable properties are.

\subsection{Enforcement of Different Modular Security Requirements}

The determination of system security by some composable property is dependent on the way of system decomposition, as pointed out earlier. In fact, this property is effective if the system is decomposed in such a way that each of its modules has quite independent security-related operations. This is because the independent operations of each module reflect certain security properties of the system, and hence they directly affect the system security.

However, for the modules which have strongly dependent security-related operations, they do not directly affect the security of the system. This implies that some insecure modules are still able to make their system meet its security requirement. For instance, the deducible property of module $M_{2}$ in the example described in Section 3.1 does not stop the system $S$ satisfying nondeducibility. In this situation, the composable property is not effective:

In practice, it is difficult to decompose a system into the modules each of which has independent security-related operations. Alternatively, the system is divided into quite large modules in order to let them have such independent operations. Consequently the advantage of security evaluation based on smaller modules is lost.

In general, operations of different modules in a system could have strong dependencies, and it is difficult for each module to possess the same security property demanded by some composable property. Thus different modules should be allowed to have different security properties or even to be insecure, provided that their composition produces a secure system. This raises the issue of whether or not the three classes of composable properties are able to handle composition of modules with different security properties.

\section{COMPARISON OF DIFFERENT COMPOSABLE PROPERTIES}

We now come to compare the three classes of composable properties with regard to the issues raised in Section 3. In the subsequent subsections our comparison is conducted based on the assumption that different composable properties are applied to assess system security with 
respect to the same security requirement.

\subsection{Generability}

We first compare the generality of composable properties of the three classes, where "generability" refers to the ability to handle various secure compositions of modules. From the classification given in Section 2, it is clear that an independent composable property is defined based mainly on internal operations of a module, while a dependent composable property is specified in connection with both internal operations of a module and its interactions with others.

In fact, the independent property is developed by assuming worst scenarios of interactions among modules. That is, the independent property can be treated as a special case of the dependent property. This means that the dependent composable property is able to deal with more cases of secure module compositions than the independent one.

Similarly the dependent composable property is considered to be a special case of a strongly dependent composable property. This is due to the fact that the dependent property requires each module to have the same security property, whereas the strongly dependent property enables different modules to have different security properties so long as their composition produces a secure system. Thus the three classes of composable properties are listed in the order of increasing generality:

\section{Independent class}

\section{Dependent class}

\section{Strongly dependent class}

This list can be used to explain the issue of system decomposition raised in Section 3.1. The independent class of composable property is very much dependent on the way in which the system is decomposed, because it has the lowest generality, i.e. it deals with only some cases of secure module compositions. If composition of modules generated in a way of system decomposition belongs to one of these cases, then it is accepted by the composable property, and not otherwise.

The strongly dependent class of composable property is nothing to do with system decompositions since it has the highest generality, i.e. it is able to handle all the cases of secure module compositions. The dependent class of composable property is related to system decompositions but not so close as the independent class.

\subsection{Security Strength}

We come to compare the security strength of the three classes of composable properties, where "security strength" means the degree of imposing over-strong security requirements on modules. As indicated above, an independent composable property is a special case of a dependent one. This implies that if a module meets the independent property, then it also meets the dependent property, but not conversely. In other words, the independent property places stronger security requirements on modules than the dependent one does. Similarly the dependent property imposes stronger security requirements on modules than a strongly dependent one. 
Hence the three classes of composable properties are named in the order of increasing security strength:

1. Strongly dependent class

\section{Dependent class}

3. Independent class

This list can be employed to explain the issues of security strength and different security requirement enforcement raised in Sections 3.2 and 3.4. First of all, let us consider modular security requirements. In general, a strongly dependent composable property places the minimum requirements on modules due to its weakest security strength.

An independent composable property imposes excessive requirements on modules because of its strongest security strength or the worst case assumption of interactions among modules. Obviously a dependent composable property is less excessive than the independent property but more than the strongly dependent one.

We then examine system security requirements. A strongly dependent composable property is able to enforce system security requirements of various strength, because this property allows the security requirement of a system to be different from those of its modules, and the property is defined specifically based on the system operations.

Nevertheless, the independent and dependent classes of composable properties can only enforce certain kinds of security requirements, since the properties demand that a system and its modules have the same security property, and they are developed with regard to general cases. Thus, for some specific system security requirement, these two classes of composable properties may be difficult to apply.

\subsection{Security Evaluation Costs}

We now proceed to compare the three classes of composable properties in connection with the cost of security evaluation. As described earlier, the security analysis of a module in terms of an independent composable property is based mainly on internal operations of the module, while the analysis of the module with regard to a dependent composable property is carried out in terms of not only internal operations of the module but also its interactions with others. The comparison of these two properties is two-fold.

On the one hand, the dependent property examines interactions of a module in conjunction with operations of other modules, whereas the independent property assesses the interactions of the module based solely on its own operations regardless of those of other modules. So the implementation of the independent property is easier than that of the dependent one in the sense of interactions. On the other hand, the dependent property imposes weaker security restrictions on the module as described in Section 4.2. This indicates that the dependent property spends less effort on security analysis than the independent one.

In general, the implementation cost of the dependent property could be higher than that of the independent one. Similarly the implementation cost of a strongly dependent composable property could be higher than that of the dependent one. That is, the three classes of composable properties can be listed in the order of increasing implementation costs: 


\section{Independent class}

\section{Dependent class}

\section{Strongly dependent class}

This list can be used to explain the issue of evaluation costs raised in Section 3.3.

\subsection{Reusability}

We finally compare the reusability of composable properties of the classes. The security analysis of a module in terms of an independent composable property is not related to operations of others, i.e. the security properties of the module do not affect those of the others. This states that if a module is modified or added to the system, then the module needs to meet the independent property so as to remain the system security property unchanged. And security reevaluation of other modules is not demanded, i.e. security properties of these other modules are reusable to deal with their composition.

In terms of a dependent composable property, nevertheless, the security evaluation of a module is associated with operations of others. This means that if a module is modified or added to the system, then security properties of other relevant modules are affected, and hence their security properties need to be re-evaluated. Clearly, the reusability of the independent property is better than that of the dependent one. Similarly the reusability of the dependent property is better than that of a strongly dependent one. Thus the three classes of composable properties are named in the order of increasing reusability:

1. Strongly dependent class

\section{Dependent class}

\section{Independent class}

The issue of security re-evaluation mentioned in Section 3.3. can be explained by this list.

\subsection{Summary}

The results described above are illustrated by the following table:

\begin{tabular}{ccccc}
\hline Class & Generality & Strength & Cost & Reusability \\
\hline Independent & 1 & 3 & 1 & 3 \\
Dependent & 2 & 2 & 2 & 2 \\
Strongly-Dep. & 3 & 1 & 3 & 1 \\
\hline
\end{tabular}

This table shows that each of the three classes of composable properties has certain advantages but also disadvantages. For instance, the advantages of independent and strongly dependent composable properties are that the implementation cost of the independent property is lowest, and the security strength of the strongly dependent property is weakest. Their disadvantages are that the security strength of the independent property is strongest, and the implementation cost of the strongly dependent one is highest. 
Note that the effects of the dependent class are between those of the independent and strongly dependent classes, as shown in the table. It is difficult to decide to what extent the dependent class is close to the strongly dependent (or independent) class. This extent is very much dependent on application systems.

\section{EFFECTIVE APPLICATION OF COMPOSABLE PROPERTIES}

From the comparison conducted in Section 4, it can be seen that each of the three classes of composable properties has certain good but also bad features. This raises the issue of how to use the composable properties in a cost-effective manner so as to retain their good features and to eliminate or reduce most of their bad features.

For convenience, let us discuss how to effectively apply independent and strongly dependent composable properties with regard to their security strength and implementation costs. To do so, a mixed version of the composable properties is probably more effective and desirable. Obviously these mixed composable properties must be consistent in the sense that if each module meets its security property, then their overall system satisfies the system security property.

An instance of such a mixed version is that the independent property is applied to analyse security properties of some modules, and the strongly dependent one to evaluate those of others. Another instance is that the independent property is employed to allocate security requirements to subsystems of a large system in a top-down way, and then the strongly dependent one to assess security properties of each subsystem based on its modules in a bottom-up manner.

One of the reasons for adopting such a mixed version is to make use of the good features of the composable properties, and to reduce their bad features. For example, this mixed version takes advantage of the strongly dependent property to place more appropriate security requirements on modules, and it cooperates with the independent property in properly reducing the implementation cost of the strongly dependent property. Thus effective trade-offs between security strength and implementation costs are made by using this method. Another reason is that this mixed version can well match system design methods since, in practice, design of a system is usually carried out in a way combining top-down and bottom-up.

However, which composable property in this mixed version should be used to analyse security properties of a specific module is very much related to concrete application. To find a solution, we carried out a case study to compare the generality of the independent and strongly dependent properties. In this case study, an overall system is supposed to be composed of only two modules. The input and output entities of the modules and their connections are the same as those shown in Fig. 1.

The study we conducted is that at each time, different functionalities are assigned to the modules, and then security properties of the modules are used to decide if the system meets nondeducibility, in terms of these two composable properties, respectively. The interesting result we obtained is that when each of entities $h_{i}^{\prime}$ and $l_{o}$ has 3 values, about $31 \%$ of secure compositions accepted by the strongly dependent property are also accepted by the independent property. When each of these entities has 10 values, the percentage dropped to about $2 \%$. That is, this proportion decreases as the domains of the entities increase. 
This case study indicates that the generality of the independent property is reduced dramatically if the numbers of input and output values are large, and consequently many secure compositions have to be wrongly rejected by this composable property. Thus, when the mixed version of the composable properties is applied, the independent property is better employed to evaluate the security of some modules that have small numbers of input and output values or quite independent security-related operations, in order to reduce the possibility of wrong rejection. And the strongly dependent property is used to decide the security of other modules.

This mixture of the composable properties enables us to properly deal with the case where modules of weak security are composed into a system of strong security. The significance of this result is that the properties can be applied to develop secure systems in a cost-effective manner. Three examples of such application are illustrated as follows. First, some existing untrusted or insecure modules or components could be re-used to build up a secure system. This can improve the reusability of the modules and reduce the cost of the system development.

Secondly, some patterns of secure compositions of modules with various security strength can be identified, each of which describes a case where modules and their connections possess special attributes. Obviously these patterns should be capable of enforcing appropriate security requirements on modules and tolerating some insecure modules. The purpose for doing so is that if attributes of modules and their connections match one of the patterns, then the pattern can ensure that the system composed of the modules is secure. Hence the security analysis of the system is simplified. This is particularly useful for design and evaluation of distributed systems.

Thirdly, the architecture of a secure system usually requires that the trusted part of the system be small enough to specify and verify formally, while the rest is assumed to be completely untrusted. This leads to the problems of bottlenecks and imposition of over-strong security requirements on the trusted part, which show some reasons for the unpopularity of the architecture. The result of our study indicates that the trusted part of the system may be unnecessary to possess strong security, and the untrusted part could enhance system security. This implies that the division of the trusted and untrusted parts is inappropriate. In fact, these two parts can cooperate to achieve system security in a better way. Thus the composable properties should be used to improve the architecture.

\section{LESSONS LEARNED}

In general, the issues of system functionality, performance, modularisation, security and costs should be taken into account in order to apply the three classes of composable properties effectively. In the following we summarise the lessons we learned from application of the composable properties, with regard to these issues.

\section{Modularise systems properly.}

To simplify security analysis, modularisation should consider the following issues:

- Security-related and security-independent functionalities are implemented in separate modules as much as possible, because the effort of security evaluation can be concentrated on these security-related modules. 
- Input and output entities of different security levels are involved in the same module as little as possible. This is due to the fact that security analysis is much easier for a module whose input and output entities have the same security level.

- Independent input or output entities are better included in separate modules, where independent input or output entities mean that each of the entities can receive any value no matter what values others have. The benefit of doing so is that the number of combinations of input or output values is reduced, and consequently the effort of security analysis is reduced.

- Shared entities such as global variables, which are used in different modules but not specified in their interfaces, are used as little as possible. This simplifies implicit dependencies among modules, and enables security analysis to be easier.

2. Apply composable properties appropriately.

An independent composable property is effective, if operations of each module are relatively independent, or the numbers of input and output values of modules are small, and the modules have a weak security requirement. This is because the possibility of wrong rejection made by this composable property can be reduced under such conditions, as indicated earlier. Otherwise a dependent or strongly dependent composable property is applied. In particular a strongly dependent composable property offers most appropriate security analysis.

\section{Reduce the cost of security evaluation.}

The three classes of composable properties can be applied according to the order in the list of implementation costs given in Section 4.3. That is, an independent composable property is first used to evaluate security properties of modules since its implementation cost is lowest. If some of the modules do not meet this property, then there are two cases to consider.

First, these modules are combined with others into bigger modules, and, as a result, these bigger modules could be able to satisfy the composable property. Secondly, functional specifications of the modules are modified to meet the composable property, provided that these modified modules still conform to the system functional specifications.

If the modules still fail to meet the composable property, then a dependent composable property is employed, and furthermore a strongly dependent composable property is used, to determine their security properties before they are finally announced to be insecure.

\section{Make rational trade-offs between security and functionality or performance.}

In practice, the security requirements of some systems are difficult to satisfy without degrading their performance or weakening their functionality. So relatively weak security requirements need to be enforced on the systems in order to make rational trade-offs between security and functionality or performance.

In the circumstances, composable properties of the independent and dependent classes may not be effective because they are logically too strong to apply. Therefore the better choice for enforcing such security requirements is composable properties of the strongly dependent class because of their generality.

5. Achieve a cost-effective method for security analysis. 
As introduced in Section 5, the three classes of composable properties can be applied in a mixed way so as to make use of their good features and to reduce their bad ones. How and to what extent the composable properties are mixed very much depend on concrete applications. In general, such a mixture should be determined in terms of dependencies among and operational properties of the modules, strength of security requirements, and cost of security evaluation.

\section{CONCLUSIONS}

In this paper we have classified and compared current composable properties. Based on our experience, we have come to feel that a single class of composable property usually does not offer a better way for handling secure composition of modules, and that a mixed version of composable properties of different classes sometimes provides a cost-effective method for secure composition. To use the different classes of composable properties appropriately, we have summarised the lessons we learned from application of these composable properties, with regard to the issues of system functionality, performance, modularisation, security and costs. We hope that the work presented in this paper can provide the material motivation for developing more useful and practical approaches to secure composition of modules.

\section{References}

1. Johnson, D.M. and Thayer, F.J. , "Security and the Composition of Machines," in Proceedings of The Computer Security Foundations Workshop, 1988.

2. Meadows, C. , "Using Traces Based on Procedure Calls to Reason About Composability," in Proc. of IEEE Symp. on Security and Privacy, 1992.

3. McCullough, D. , “A Hookup Theorem for Multilevel Security," IEEE Trans., Software Eng., vol. SE-16, no. 6, June 1990.

4. McDermid, J.A. and Shi, Q. , "Secure Composition of Systems," in Proceedings of the Eighth Annual Computer Security Applications Conference, IEEE Computer Society Press, December 1992.

5. Millen, J.K. , "Hookup Security for Synchronous Machines," in Proceedings of The Computer Security Foundations Workshop, 1990.

6. Shi, Q. , McDermid, J.A. , and Moffett, J.D. , "Developing Secure Systems in A Modular Way," in Proceedings of the Eighth Annual Conference on Computer Assurance, IEEE and BCS, June 1993.

7. Shi, Q. , McDermid, J.A. , and Moffett, J.D. , “Applying Noninterference to Composition of Systems: A More Practical Approach," in Proceedings of the Ninth Annual Computer Security Applications Conference, IEEE Computer Society Press, December 1993.

8. Shi, Q. and McDermid, J.A. , "Constructing Secure Distributed Systems Using Components," in Proceedings of the 12th Symposium on Reliable Distributed Systems, IEEE Computer Society Press, October 1993.

9. Boulahia-Cuppens, N. and Cuppens, F., "Asynchronous Composition and Required Security Conditions," in Proc. of IEEE Symp. on Security and Privacy, 1994. 
10. Guttman, J.D. and Nadel, M.E., "What Needs Securing?," in Proceedings of the Computer Security Foundations Workshop, IEEE Computer Society Press, June 1988.

11. McLean, J., "A General Theory of Composition for Trace Sets Closed Under Selective Interleaving Functions," in Proc. of IEEE Symp. on Security and Privacy, 1994.

12. Wittbold, J.T. and Johnson, D.M. , "Information Flow in Nondeterministic Systems," in Proc. of IEEE Symp.on Security and Privacy, May 1990. 